\section{Differences between the gut microbial composition of diarrheic and healthy dogs}

\section{Ronnie Gueta, Tamara Wunder, Anton Heusinger, Elisabeth Müller}

Laboklin GmbH \& Co. KG, Bad Kissingen, Germany

\section{OBJECTIVES}

Imbalances in the microbial composition of the intestinal microbiome are associated to various gastrointestinal diseases like chronic inflammatory enteropathy (CED) or inflammatory bowel disease (IBD). Recent studies have identified eight bacterial groups that are altered in dogs with chronic or acute gastrointestinal signs in comparison to healthy individuals and may be used as biomarkers for dysbiosis. Aim of the study was to establish a method suitable for the routine diagnostic screening of the intestinal microbiota for dysbiotic changes in dogs.

\section{METHODS}

DNA from fecal samples from dogs $(n=10)$ with acute diarrhea and healthy dogs $(n=10)$ as control group was extracted during veterinary routine diagnostics. Subsequently qPCR assays targeting 16S rRNA genes for eight different bacterial groups (Faecalibacterium prausnitzii, Fusobacteria, Blautia, Turicibacter, Escherichia coli, Clostridium hiranonis, Streptococcus and total bacteria) were performed and total copy numbers per $\mathrm{g}$ faeces calculated.

\section{RESULTS}

The intestinal microbiota of dogs with acute diarrhea were different to the one of the healthy control group. Particularly F. prausnitzii, an anaerobic species that has been reported to have beneficial effects on the integrity of the intestinal mucosal barrier was decreased 5 -fold. In contrast, proteolytic microorganisms that may induce intestinal tumorogenesis or have pro-inflammatory potential like Fusobacteria were increased up to 10 -fold.

\section{STATEMENT (CONCLUSIONS)}

This study supports the hypothesis that alterations of the intestinal microbiota correlate with acute enteropathies in dogs. Furthermore, we highlight the additional insights this type of analysis offers in the future for differentialdiagnostic purposes.

\section{Suspected Choledochal Cyst in a DSH cat}

\section{Konstantinos Fontas}

Downs Veterinary Referrals, Bristol, United Kingdom

\section{OBJECTIVES}

To present an uncommon cystic disease of the biliary tract in a cat.

\section{METHODS}

A 9-year old female neutered cat was presented with polyphagia, weight loss, urinating in the house and behaviour change. In the clinical examination, jaundice and a palpable mass in the cranial abdomen were found. Haematology and biochemistry showed increased liver enzymes, total bilirubin and leucocytosis. Abdominal radiology and ultrasonography confirmed a large cystic structure caudal to the liver. Also, ultrasound-guided needle aspiration was taken, revealing a highly proteinaceous fluid with mixed bacterial growth. After stabilization, exploratory laparotomy was performed, revealing a large, thick, fluid-filled structure within the cranial abdomen. The gall bladder and the pancreas were oedematous. Examination within the cyst showed potential communications with the duodenum and the biliary tree. Therapeutically, subtotal resection of the cyst wall was performed and after closing the connections, the remaining cavity was omentalised. Histopathology samples revealed multi-loculated cystic structure, chronic cholangitis and lymphoplasmacytic pancreatitis Postoperatively, ursodeoxycholic acid, antioxidants and antibiotics were administered .

\section{RESULTS}

The cat had an unremarkable recovery and so far the prognosis appears to be favourable. However, close monitoring is indicated to prevent recurrent biliary obstruction and ongoing hepatic and pancreatic disease.

\section{STATEMENT (CONCLUSIONS)}

A choledochal cyst is rare in the cat. In the current case, the cyst was possibly of congenital origin and gradual distension resulted in late onset clinical signs due to biliary obstruction and bacterial infection from the intestine. Furthermore, late onset cyst development can be associated with underlying intestinal, pancreatic or biliary pathology. 